\title{
Article
}

\section{Rheology of Aqueous Solution of Hydrophobically Modified Ethoxylated Urethane (HEUR) with Fluorescent Probes at Chain Ends: Thinning Mechanism}

\author{
Shinya SUZUKI ${ }^{*, * *}$, Takashi UnEYAMA ${ }^{*}$, Tadashi INOUE ${ }^{* * *}$, and Hiroshi WATANABE ${ }^{*, \dagger}$ \\ "Institute for chemical Research, Kyoto University, Uji, Kyoto 611-0011, Japan \\ ${ }^{* *}$ Lintec corporation, 5-14-42 Nishikicho, Warabi, Saitama 335-0005, Japan \\ ****Department of Macromolecular Science, Graduate school of Science, Osaka University, \\ Toyonaka, Osaka 560-0043, Japan \\ (Received : December 7, 2011)
}

\begin{abstract}
Nonlinear flow behavior and the corresponding fluorescent behavior were examined for a $3.0 \mathrm{wt} \%$ aqueous solution of hydrophobically modified ethoxylated urethane (HEUR; $M_{\mathrm{w}}=1.1 \times 10^{5}$ ) having fluorescent pyrenyl (Py) groups at the chain ends. These end groups associated with each other to serve as cores of HEUR flower micelles, and these micelles were further connected into super-bridges to form a transient network. Correspondingly, single-Maxwellian relaxation reflecting the thermal reorganization of this network was observed in the linear viscoelastic regime. Under steady shear flow, the HEUR solution exhibited thinning of both viscosity $\eta(\dot{\gamma})$ and first normal stress coefficient $\Psi_{1}(\dot{\gamma})$ at shear rates $\dot{\gamma}$ above the equilibrium relaxation frequency $1 / \tau$. Fluorescent emission intensity $I_{\mathrm{E}}(\dot{\gamma})$ from excimers formed by Py groups associated in a hydrophobic environment of the micellar cores and the intensity $I_{\mathrm{M}}(\dot{\gamma})$ from dissociated Py groups (referred to as monomers) isolated in the aqueous phase were measured under flow simultaneously with $\eta$ and $\Psi_{1}$. The $I_{\mathrm{E}} / I_{\mathrm{M}}$ ratio was found to decrease only slightly (by a factor of $\sim 4 \%$ ) on an increase of $\dot{\gamma}$ up to $6 / \tau$ well in the thinning regime. (For $\dot{\gamma}=6 / \tau, \eta$ and $\Psi_{1}$ decreased from respective zero-shear values by factors of $\sim 50 \%$ and $\sim 75 \%$, respectively) Thus, the significant thinning at those $\dot{\gamma}$ was accompanied by a negligible change in the HEUR core structure that corresponds to just a slight shift of the association/dissociation balance of the Py groups to the dissociated state. From this result, the thinning is quite possibly attributed to flow-induced disruption of the network connectivity through conversion of the super-bridges into super-loops, both having the same core structure.
\end{abstract}

Key Words: Hydrophobically modified ethoxylated urethane (HEUR) / Flower micelle / Shear thinning /

Fluorescent emission / Flow-induced network disruption

\section{INTRODUCTION}

Hydrophobically-modified ethoxylated urethane (HEUR) is a representative water-soluble telechelic polymers. ${ }^{1-6)}$ HEUR is composed of poly(ethylene oxide) (PEO) main chain and short hydrophobic end groups such as alkyl groups, the latter associating with each other in aqueous solutions to bind the PEO chains into flower micelles. ${ }^{5,6)}$ At high concentrations $c$, some micelles are connected into strings (through HEUR chains having the end groups in different cores) and these strings, often referred to as super-bridges, ${ }^{5,6)}$ are organized into a transient network spreading throughout the whole solution.

Slow rheological responses of high-c HEUR $a q$ solutions are governed by this transient network. ${ }^{1-23)}$ For example,

$\dagger$ to whom the correspondence should be addressed these solutions exhibit the single-Maxwellian relaxation in the linear viscoelastic regime, as first noted by Annable et al. ${ }^{5,6)}$ This relaxation behavior reflects the thermal reorganization (dissociation/association) of the HEUR network, and the relaxation time corresponds to the lifetime of the HEUR core acting as the physical crosslink for the network. The relaxation time and the viscosity of the HEUR $a q$ solutions change with temperature, concentration, and type of the end groups, as revealed in extensive studies. ${ }^{7-10)}$ Furthermore, optical experiments ${ }^{20-22)}$ have been made for solutions of HEUR chains containing fluorescent pyrenyl (Py) groups, either chemically attached to the chain $e^{20)}$ or just dissolved in the solutions, ${ }^{13,21,22)}$ to detect the fluorescent emission from excimers formed by the Py groups associated in the hydrophobic cores. We may utilize the fluorescent emission data to estimate, for example, the fraction of dangling chain ends in solutions. ${ }^{13)}$ Those data, if combined 
with some statistical models, may also give an estimate of the aggregation number (the average number of chain ends per micellar core). ${ }^{13,20)}$

Under fast shear flow, the HEUR aq solutions exhibit significant nonlinear behavior, thickening and thinning of the steady state viscosity $\eta,^{3,5,9,11,14-19,20,22,23)}$ which again reflects the transient nature of the HEUR network. Specifically, the thickening often noted at shear rates $\dot{\gamma}$ just above the equilibrium relaxation frequency $1 / \tau$ has been attributed to the finite extensible nonlinear elasticity (FENE) of the HEUR strands under fast shear, 3,14-16,24,25) or, the shear-induced increase of the number density $v$ of effective bridge chains. ${ }^{17-19)}$ However, recently, both $\eta$ and the first normal stress coefficient $\Psi_{1}$ were examined in the transient as well as steady flow states to reveal that the thickening of $\eta$ of a model HEUR solution is accompanied with no nonlinearity of $\Psi_{1}{ }^{23)}$ This lack of the nonlinearity of $\Psi_{1}$ does not necessarily match with the conventionally considered mechanisms, FENE and shear-induced increase of $v$, thereby suggesting a delicate but realistic mechanism of thickening, anisotropic creation of the bridges under shear preferentially occurring in the shear gradient direction (and being in balance with the shear-induced rupture of the bridges). ${ }^{23)}$

Differing from the behavior explained above, the thinning of $\eta$ seen at $\dot{\gamma} \gg 1 / \tau$ is accompanied by thinning of $\Psi_{1}{ }^{23)}$ which suggests that the thinning reflects the shearinduced disruption of the HEUR network (decrease of $v) .^{3,5,9,11,14-20,22,23)}$ This assignment of the thinning mechanism sounds reasonable but, to our best knowledge, the shearinduced disruption has not been observed directly, except in one paper reporting fluorescent behavior of the Pytagged HEUR chains under flow. ${ }^{20)}$ Even in that paper, the $\eta$ and $\Psi_{1}$ data were not well analyzed in relation to the fluorescent data.

Thus, we conducted rheological and optical experiments for a model HEUR chain having Py probes at the chain end to examine the structural origin of shear thinning of $\eta$ and $\Psi_{1}$ at $\dot{\gamma} \gg 1 / \tau$. It turned out that the fluorescent emission intensity from the excimers of Py groups (in the cores) decreases only slightly on an increase of $\dot{\gamma}$ up to $6 / \tau$ well in the thinning regime. This result suggested that the thinning of $\eta$ and $\Psi_{1}$ is mostly due to shear-induced disruption of the network connectivity through conversion of the super-bridges into super-loops, both having the same structure of the cores and thus exhibiting the same fluorescent behavior. Details of these results are presented in this paper.

\section{EXPERIMENTAL}

\subsection{Material}

HEUR having butylpyrenyl (Bu-Py) groups at the chain ends, as illustrated in Figure 1, was synthesized with a standard method. ${ }^{12)}$ The chemicals utilized in the synthesis, poly(ethylene oxide) $\left(\mathrm{PEO} ; M_{\mathrm{w}}=1.9 \times 10^{4}, M_{\mathrm{w}} / M_{\mathrm{n}}=1.1\right)$ and methylene diphenyl-4,4'-diisocyanate (MDPDI), were purchased from Wako Pure Chemical Industries Ltd, and 1-pyrenyl butanol (Py-BuOH), from Sigma-Aldrich Co. All these chemicals were used without further purification. The synthesis was conducted in dehydrated tetrahydrofuran (THF; Guaranteed grade, Wako) containing $25 \mathrm{wt} \%$ of PEO and given masses of $\mathrm{Py}-\mathrm{BuOH}$ and MDPDI (molar ratio PEO:MDPDI:Py-BuOH = 3:4:2). The PEO chains were first extended through the condensation reaction with MDPDI at $60{ }^{\circ} \mathrm{C}$ for $2 \mathrm{~h}$, and then the end-capping reaction between Py$\mathrm{BuOH}$ and MDPDI, the latter remaining active at the ends of the extended PEO chains, was conducted at $60{ }^{\circ} \mathrm{C}$ for $24 \mathrm{~h}$. Then, the reaction mixture was cooled to room temperature, diluted with excess THF, and poured into a large volume of $\mathrm{THF} /$ hexane mixture of $1 / 3$ (wt/wt) composition to recover the HEUR sample as a precipitant. The sample was thoroughly dried in vacuum at $40{ }^{\circ} \mathrm{C}$.

The HEUR sample was characterized with size-exclusion chromatography utilizing a column/pump system (HLC8320 GPC EcoSEC, Tosoh) equipped with a refractive index monitor. The elution solvent was THF, and commercially available monodisperse PEO samples (Tosoh) were utilized as the solution standards. The weight average molecular weight and polydispersity index of the HEUR sample, determined from the elution volume calibration with those standards, were $M_{\mathrm{w}}=1.1 \times 10^{5}$ and $M_{\mathrm{w}} / M_{\mathrm{n}}=1.7_{8}$, respectively.

The material subjected to the rheological measurements was a $3.0 \mathrm{wt} \%$ aqueous solution of the HEUR sample in distilled water. Prescribed masses of water and HEUR were stirred for 24 hours to prepare the solution.

\subsection{Measurements}

For the $3.0 \mathrm{wt} \%$ aqueous solution of HEUR, rheological and fluorescent measurements were conducted with a laboratory rheometer, MCR-301 (Anton Paar).

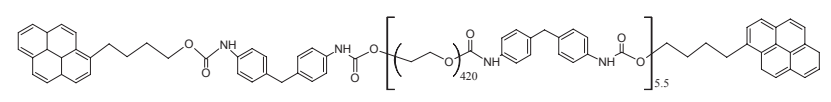

Fig. 1. Structure of HEUR chains having pyrenyl groups at both ends. 
Dynamic measurements in the linear viscoelastic regime were conducted with a cone-plate (CP) fixture (made of SUS304 stainless steel) having a diameter $d=75 \mathrm{~mm}$ and a cone angle $\theta=1.0^{\circ}$. The storage and loss moduli, $G^{\prime}(\omega)$ and $G^{\prime \prime}(\omega)$, were measured as functions of the angular frequency $\omega$ at several temperatures between 5 and $25^{\circ} \mathrm{C}$. Those data obeyed the time-temperature superposition at low $\omega$ where the HEUR network exhibited the terminal relaxation (through its thermal reorganization). The data were reduced at $5{ }^{\circ} \mathrm{C}$.

The viscosity and first normal stress coefficient in the steady state, $\eta(\dot{\gamma})$ and $\Psi_{1}(\dot{\gamma})$, were measured at $5{ }^{\circ} \mathrm{C}$ as a function of the shear rate $\dot{\gamma}$. (The relaxation of the HEUR solution was too fast to allow accurate measurements of the transient, viscosity and normal stress growth functions.) The measurements were made with two sets of CP fixture, one with $d=75 \mathrm{~mm}$ and $\theta$ $=1.0^{\circ}$ (the fixture explained above), and the other with $d=40$ $\mathrm{mm}$ and $\theta=5.7^{\circ}$ made of quartz.

The latter set of CP was utilized to measure, simultaneously with $\eta(\dot{\gamma})$ and $\Psi_{1}(\dot{\gamma})$, the fluorescent emission intensity $I_{\mathrm{E}}(\dot{\gamma})$ from the excimers of Py groups associated in the hydrophobic cores of the HEUR micelles. Ultraviolet (UV) light of the wavelength $\lambda=340 \mathrm{~nm}$ (fluorescent excitation trigger), obtained from a UV lamp (Max-301; Asahi Spectra), was introduced to this quartz CP fixture, and the fluorescent emission spectra were measured in a range of $\lambda=350 \mathrm{~nm}$ $650 \mathrm{~nm}$ with a spectrometer (PAM-11; Hamamatsu Photonics). Some Py groups transiently dissociate from the core and are isolated in the aqueous phase. The fluorescent emission from these dissociated Py groups (referred to as monomers) was observed at $\lambda=370-410 \mathrm{~nm}$, and that from the Py excimer was seen at $\lambda=410-650 \mathrm{~nm}^{21,22)}$ The intensities of the monomer and excimer emission, $I_{\mathrm{M}}(\dot{\gamma})$ and $I_{\mathrm{E}}(\dot{\gamma})$, were measured as peak intensities at $\lambda=377 \mathrm{~nm}$ and $487 \mathrm{~nm}$, respectively, and their ratio, $I_{\mathrm{E}}(\dot{\gamma}) / I_{\mathrm{M}}(\dot{\gamma})$, was utilized as a measure of the number of the Py groups in the HEUR cores. (Note that a slight fluctuation of the UV lamp intensity was canceled in this ratio.)

\section{RESULTS}

\subsection{Linear Viscoelastic Behavior}

For the $3.0 \mathrm{wt} \%$ aqueous solution of HEUR, the storage and loss moduli data, $G^{\prime}(\omega)$ and $G^{\prime \prime}(\omega)$, measured at several temperatures $\left(5-25^{\circ} \mathrm{C}\right)$ obeyed the time-temperature superposition (tTS) in the range of $\omega$ examined. Figure 2 shows the master curves of those data reduced at $T_{\mathrm{r}}=5^{\circ} \mathrm{C}$. The solid curves indicate the result of fitting with the singleMaxwellian model:

$$
G^{\prime}(\omega)=G_{0} \frac{\omega^{2} \tau^{2}}{1+\omega^{2} \tau^{2}}, \quad G^{\prime \prime}(\omega)=G_{0} \frac{\omega \tau}{1+\omega^{2} \tau^{2}}
$$

Here, $G_{0}(=310 \mathrm{~Pa})$ and $\tau(=0.022 \mathrm{~s})$ are the high frequency plateau modulus and relaxation time, respectively. The fit is excellently achieved at low $\omega$, as noted also for similar HEUR solutions. ${ }^{5,6,23)}$ A similar HEUR solution ${ }^{23)}$ exhibited the Maxwellian terminal relaxation obeying tTS but also the relaxation within the HEUR strand at high $\omega$ (not examined for our HEUR solution in Figure 2). This intra-strand relaxation gives a non-Maxwellian character to the solution and disturbs tTS. A small deviation from the Maxwellian fitting seen in Figure 1 can be attributed to the intra-strand relaxation.

The single-Maxwellian terminal behavior of the HEUR solution is attributed to the thermal reorganization of the transient network occurring at the time $\tau$. This reorganization occurs through dissociation/association of the Bu-Py groups at the HEUR chains, and the interior methylene diphenyl-4,4'diisocyanate groups (MDPDI; cf. Figure 1) are polar enough to exhibit no association, as suggested from the behavior of a similar HEUR chain having the interior MDPDI groups and end-capping hexadecyl groups. ${ }^{23)}$ Consequently, the plateau modulus $G_{0}$ prevailing at high $\omega$ (before the dissociation) is related to the entropy elasticity of the active strands, and the number density $v$ of those strands is estimated as

$$
v=\frac{G_{0}}{k_{\mathrm{B}} T} \cong 8.1 \times 10^{22} \mathrm{~m}^{-3}
$$

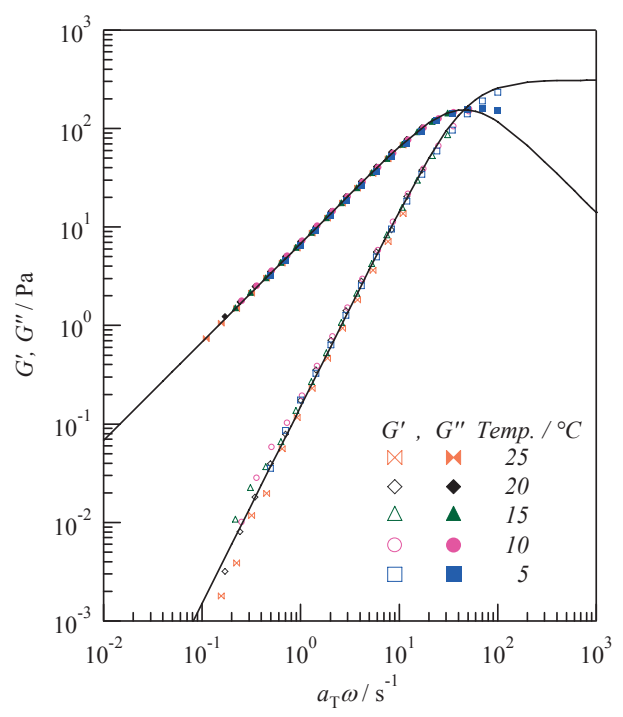

Fig. 2. Master curves of storage and loss moduli of the $3.0 \mathrm{wt} \%$ HEUR $a q$ solution reduced at $5{ }^{\circ} \mathrm{C}$.

The solid curves indicate the results of fitting with singleMaxwellian behavior. 
Here, $k_{B}$ is the Boltzmann constant and $T$ is the absolute temperature. This $v$ value is smaller than the number density of the HEUR chains, $v_{\mathrm{o}}=2.9 \times 10^{23} \mathrm{~m}^{-3}$ evaluated from the HEUR concentration and molecular weight, $c=0.03 \mathrm{~g} \mathrm{~cm}^{-3}$ and $M_{\mathrm{n}}=6.2 \times 10^{4}$. (The $v / v_{\mathrm{o}}$ ratio $(=0.28)$ of our HEUR chains having butylpyrenyl groups at the ends is similar to the $v / v_{\mathrm{o}}$ ratio $(=0.15)^{13}$ noted for $0.027 \mathrm{~g} \mathrm{~cm}^{-3}$ solution of HEUR chains having hexadecyl groups.) The functionality $f$ of the network (the average number of elastically active chains per micellar core) is roughly estimated to be $f \approx n v / v_{0}$ (where $n$ is the association number). ${ }^{13)}$ For our HEUR solution, the functionality is estimated as $f \approx 1.6$ (with $\mathrm{n} \approx 6$ estimated by the fluorescent data ${ }^{20)}$ ), which is not large enough to form a densely interconnected network. This result suggests that the bridged sequence (string) of the flower micelles should behave as the active strands, although some fraction of those strings would be of loop-type and not involved in the active strands.

Figure 3 shows the temperature dependence of the shift factor $a_{\mathrm{T}}$ for the master curves of $G^{\prime}$ and $G^{\prime \prime}$. The Arrhenius behavior, $\ln a_{\mathrm{T}}=E_{\mathrm{a}}\left(T^{-1}-T_{\mathrm{r}}^{-1}\right) / R$ with $R$ being the gas constant, is clearly noted, and the activation energy $E_{\mathrm{a}}$ is evaluated to be $\cong 87 \mathrm{~kJ} \mathrm{~mol}^{-1}$. This $E_{\mathrm{a}}$ value can be assigned as the association energy of the butylpyrenyl groups at the HEUR chain ends that stabilizes the HEUR network in our solution. This activation energy is of the same order as the activation energies reported for HEUR chains with hexadecyl end groups. ${ }^{5,8,23)}$ From the energy competition argument based on the $E_{\mathrm{a}}$ value, ${ }^{23)}$ the nonlinear elasticity (FENE) effect is expected to be negligible in our HEUR solution.

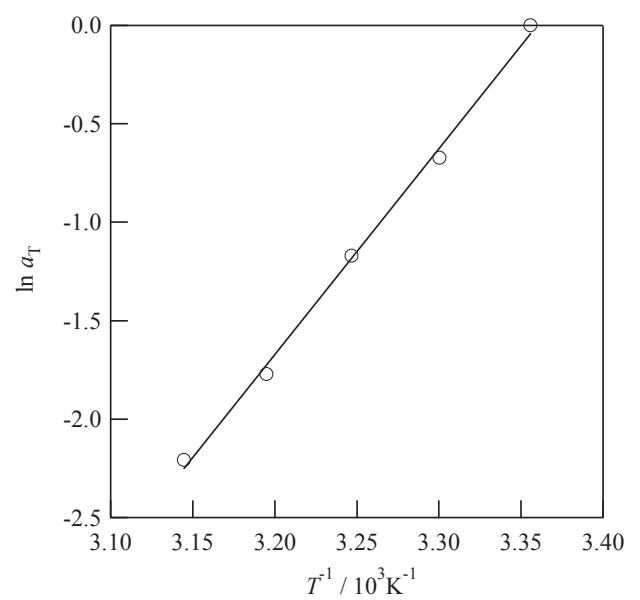

Fig. 3. Temperature dependence of the shift factor for the master curves shown in Figure 2.

\subsection{Nonlinear Flow and Fluorescent Behavior}

Figure 4 shows the steady state viscosity $\eta(\dot{\gamma})$ and the first normal stress coefficient $\Psi_{1}(\dot{\gamma})$ measured for the $3.0 \mathrm{wt} \%$ HEUR solution at $5{ }^{\circ} \mathrm{C}$. The data obtained with the coneplate (CP) fixtures with the diameter $d=75 \mathrm{~mm}$ and the cone angle $\theta=1.0^{\circ}$ (large unfilled symbols) and with $d=40 \mathrm{~mm}$ and $\theta=5.7^{\circ}$ (small filled symbols) agree with each other. The horizontal dashed lines indicate respective zero-shear values, $\eta_{0}\left(=\left[G^{\prime \prime} / \omega\right]_{\omega \rightarrow 0}\right)$ and $\Psi_{1,0}\left(=2 \eta_{0}^{2} J_{\mathrm{e}}=2\left[G^{\prime} / \omega^{2}\right]_{\omega \rightarrow 0}\right)$, obtained from the linear viscoelastic $G^{\prime}$ and $G^{\prime \prime}$ data.

The CP fixture with $d=40 \mathrm{~mm}$ and $\theta=5.7^{\circ}$ (made of quartz) was utilized to measure, under flow (together with the rheological data), the intensity of the fluorescent emission from the pyreneyl (Py) groups at the HEUR chain ends. A typical example of the emission spectra is shown in Figure 5, where $I_{\mathrm{M}}$ shows the emission from the isolated Py group (monomer) transiently dissociating from the micellar core into the aqueous phase, and $I_{\mathrm{E}}$, the emission from the excimer of the Py groups associated in the hydrophobic core. These $I_{\mathrm{M}}(\dot{\gamma})$ and $I_{\mathrm{E}}(\dot{\gamma})$ are quantified as the emission peak intensity at $\lambda=$ $377 \mathrm{~nm}$ and $487 \mathrm{~nm}$, respectively, and the spectra shown in Figure 5 have been normalized by $I_{\mathrm{M}}(\dot{\gamma})($ at $\lambda=377 \mathrm{~nm})$ so as to cancel a slight fluctuation of the excitation UV intensity. A ratio, $I_{\mathrm{E}}(\dot{\gamma}) / I_{\mathrm{M}}(\dot{\gamma})$, is utilized as a measure of the number of the Py groups associated in the HEUR cores. Figure 6 shows changes of the $I_{\mathrm{E}}(\dot{\gamma}) / I_{\mathrm{M}}(\dot{\gamma})$ ratio with the shear rate $\dot{\gamma}$.

As noted in Figure 4, the HEUR solution exhibits significant decreases of both $\eta(\dot{\gamma})$ and $\Psi_{1}(\dot{\gamma})$, by factors of $\sim 50 \%$ and $\sim 75 \%$ respectively, on an increase of the shear rate well above the equilibrium relaxation frequency $1 / \tau\left(=45 \mathrm{~s}^{-1}\right.$ determined

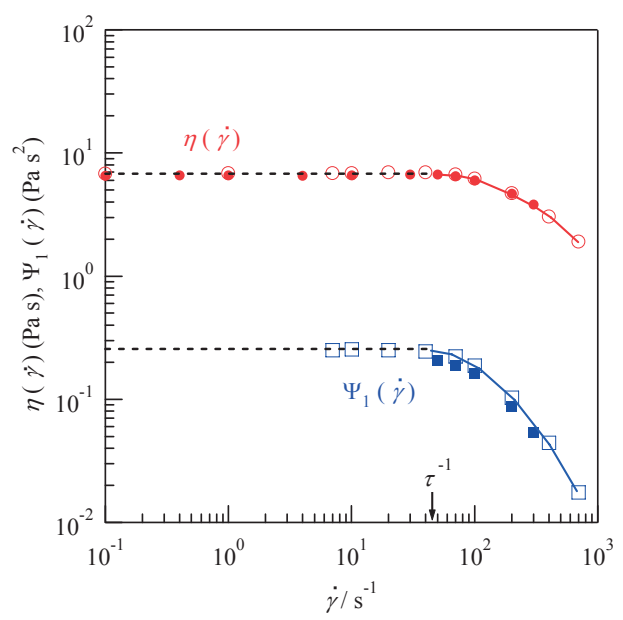

Fig. 4. Steady state viscosity and first normal stress coefficient measured for the $3.0 \mathrm{wt} \%$ HEUR $a q$ solution at $5{ }^{\circ} \mathrm{C}$. The horizontal dashed lines indicate the zero-shear values, $\eta_{0}$ and $\Psi_{1,0}\left(=2 \eta_{0}^{2} J_{\mathrm{e}}\right)$, evaluated from the linear viscoelastic $G^{\prime}$ and $G^{\prime \prime}$ data. 
from the linear viscoelastic data shown in Figure 2). Although not shown here, these $\eta(\dot{\gamma})$ and $\Psi_{1}(\dot{\gamma})$ data do not obey the empirical Cox-Merz, ${ }^{26)}$ Gleissle, ${ }^{27)}$ and Osaki ${ }^{28)}$ rules valid for homogeneous, entangled polymer systems, as noted also for other HEUR solutions. ${ }^{11,23)}$ This thinning behavior is associated with just a slight decrease (by a factor of $\sim 4 \%$ ) of the $I_{\mathrm{E}}(\dot{\gamma}) /$ $I_{\mathrm{M}}(\dot{\gamma})$ ratio (cf. Figure 6 ), which provides us with a clue for specifying the thinning mechanism, as discussed below.

At equilibrium, the Py groups are dynamically associating in/dissociating from the HEUR cores, thereby allowing the thermal reorganization of the HEUR network that results in the Maxwellian terminal relaxation in the linear viscoelastic regime. This association/dissociation balance hardly changes even under fast flow at $\dot{\gamma}=6 / \tau$, as suggested from the $I_{\mathrm{E}}(\dot{\gamma}) / I_{\mathrm{M}}(\dot{\gamma})$ data. Thus, the significant thinning seen at those $\dot{\gamma}$ is accompanied by an almost negligible change in the HEUR core structure (that corresponds to a very slight shift of the association/dissociation balance of the Py groups to the dissociated state). From this result, the thinning is quite possibly attributed to flow-induced disruption of the network connectivity through conversion of the super-bridges into super-loops, both having the same core structure. This result lends experimental support to the conventional argument for the thinning mechanism.

Finally, we remember that HEUR chains with hexadecyl (HD) groups often exhibit thickening of $\eta$ prior to the thinning. ${ }^{11,23)}$ In contrast, no thickening is observed for our HEUR chains having butyl-pyrenyl (BuPy) groups at the ends;

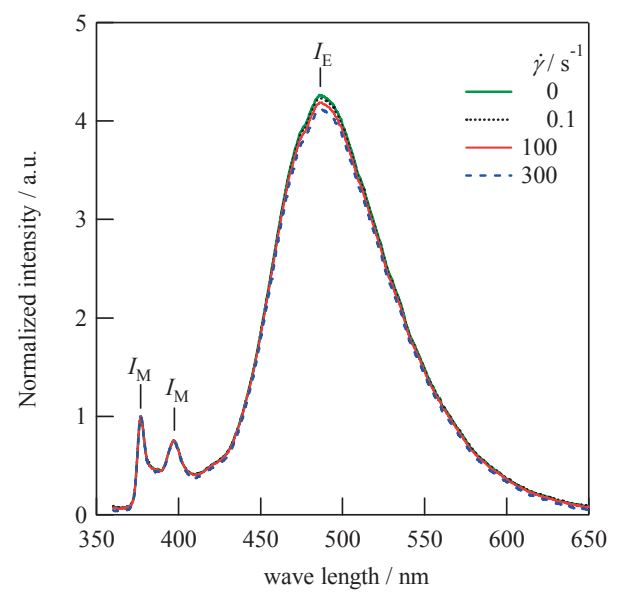

Fig. 5. Intensity of fluorescent emission from the $3.0 \mathrm{wt} \%$ HEUR $a q$ solution in the quiescent state $(\dot{\gamma}=0)$ and under steady shear flow at $5{ }^{\circ} \mathrm{C} . I_{\mathrm{M}}$ and $I_{\mathrm{E}}$ indicate the emission from the pyrenyl group (attached at the chain ends) in the isolated (monomer) state in the aqueous phase and in the excimer state in the associated HEUR cores, respectively. The spectra shown here have been normalized by $I_{\mathrm{M}}$ at $\lambda=377 \mathrm{~nm}$ so as to cancel a slight fluctuation of the excitation UV intensity. cf. Figure 4. This difference may be attributed to a difference of the number $n$ of the end groups per HEUR core: $n$ has been reported to be $\sim 22$ and $\sim 6$, respectively, for the HEUR chains having the $\mathrm{HD}^{13)}$ and $\mathrm{BuPy}^{20)}$ groups at the chain ends. It appears that the bridges sustaining the high viscosity in the thickening regime can be easily disrupted (converted to the loops) for small $n$, thereby resulting in the lack of thickening for our HEUR chains having the BuPy groups.

\section{CONCLUDING REMARKS}

For the $3.0 \mathrm{wt} \%$ aqueous solution of HEUR chains having butyl-pyrenyl groups at the ends, linear and nonlinear viscoelastic behavior was examined. The HEUR chains formed a transient network sustained by cores of associating end groups. The single-Maxwellian terminal relaxation reflecting the thermal reorganization of this network was observed in the linear viscoelastic regime. Under steady shear flow, the HEUR solution exhibited thinning of both viscosity $\eta(\dot{\gamma})$ and first normal stress coefficient $\Psi_{1}(\dot{\gamma})$ at shear rates $\dot{\gamma}$ above the equilibrium relaxation frequency $1 / \tau$. Fluorescence experiment suggested that the HEUR core structure is hardly affected even under fast shear in the thinning regime. Thus, the thinning of $\eta$ and $\Psi_{1}$ is most likely due to flow-induced disruption of the network connectivity through conversion of the super-bridges into super-loops, both having the same core structure.

\section{Acknowledgment}

This work was supported by the Grant-in-Aid for Scientific Research (B) (grant \# 21350063) and by Grant-in-Aid for Young Scientists (B) from MEXT (grant \#22740273).

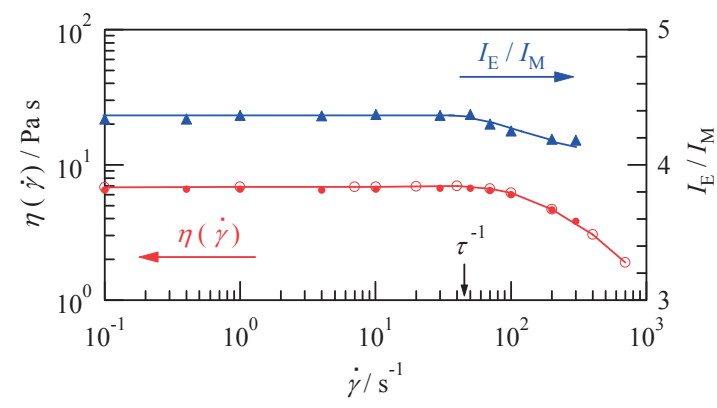

Fig. 6. Fluorescent emission intensity $I_{\mathrm{E}}$ from excimer of associated pyrenyl groups at the HEUR chain ends in the $3.0 \mathrm{wt} \% a q$ solution under steady shear at $5{ }^{\circ} \mathrm{C}$. This $I_{\mathrm{E}}$ is normalized by the emission intensity $I_{\mathrm{M}}$ from pyrenyl groups in the isolated monomer state in the aqueous phase and plotted against shear rate. For comparison, the shear rate dependence of the viscosity is also shown. 


\section{REFERENCES}

1) Lundberg DJ, Glass JE, Eley RR, J Rheol, 35, 1255 (1991).

2) $\mathrm{Xu}$ B, Li L, Yekta A, Masoumi Z, Kanagalingam S, Winnik MA, Zhang K, Macdonald PM, Langmuir, 13, 2447 (1997).

3) Berret JF, Séréro Y, Winkelman B, Calvet D, Collet A, Viguior M, J Rheol, 45, 477 (2001).

4) Calvet D, Collet A, Viguier M, Berret J, Séréro Y, Macromolecules, 36, 449 (2003).

5) Annable T, Buscall R, Ettelaie R, Whittlestone D, J Rheol, 37, 695 (1993).

6) Annable T, Buscall R, Ettelaie R, Colloids Surf A, 112, 97 (1996).

7) May R, Kaczmarski JP, Glass JE, Macromolecules, 29, 4745 (1996).

8) Kaczmarski JP, Tarng MR, Ma Z, Glass JE, Colloids Surf A, 147, 39 (1999).

9) Ma SX, Cooper SL, Macromolecules, 34, 3294 (2001).

10) Barmar M, Ribitsch V, Kaffashi B, Barikani M, Sarreshtehdari M, Pfragner J, Colloid Polym Sci, 282, 454 (2004).

11) Pellens L, Corrales RG, Mewis J, J Rheol, 48, 379 (2004).

12) Kaczmarski JP, Glass JE, Langmuir, 10, 3035 (1994).

13) Xu B, Yekta A, Li L, Masoumi Z, Winnik MA, Colloid Polym Sci A, 112, 239 (1996).

14) Berret JF, Séréro Y, Phys Rev Lett, 87, 048303-1 (2001).

15) Pellens L, Vermant J, Mewis J, Macromolecules, 38, 1911 (2005).
16) Pellens L, Ahn KH, Lee SJ, Mewis J, J Non-Newtonian Fluid Mech, 121, 87 (2004)

17) Tripathi A, Tam KC, Mckinley GH, Macromolecules, 39, 1981 (2006).

18) Tam KC, Jenkins RD, Winnik MA, Bassett DR, Macromolecules, 31, 4149 (1998).

19) François J, Maitre $S$, Rawiso $M$, Sarazin D, Beinert G, Isel F, Colloid Surf A, 112, 251 (1996).

20) Richey B, Kirk AB, Eisenhart EK, Fitzwater S, Hook J, $J$ Coating Technol, 63, 31 (1991).

21) Yekta A, Duhamel J, Brochard P, Adiwidjaja H, Winnik MA, Macromolecules, 26, 1829 (1993).

22) Yekta A, Xu B, Duhamel J, Adiwidjaja H, Winnik MA, Macromolecules, 28, 956 (1995).

23) Suzuki S, Uneyama T, Inoue T, Watanabe H, Macromolecules, in press.

24) Koga T, Tanaka F. Macromolecules, 43, 3052 (2010).

25) Koga T, Tanaka F, Kaneda I, Winnik FM, Langmuir, 25, 8626 (2009).

26) Cox WP, Merz EH, J Polym Sci, 28, 619 (1958).

27) Gleissle $\mathrm{W}$, Two time-shear rate relations combining viscosity and first normal stress coefficient in the linear and nonlinear flow range, 8th Int. Cong. Rheol., Naples, 1980.

28) Osaki K, Watanabe H, Inoue T, Nihon Reoroji Gakkaishi $(J$ Soc Rheol Japan), 26, 49 (1998). 